\title{
Known Unknowns: Representing Uncertainty in Historical Time
}

\author{
Florian Kräutli and Stephen Boyd Davis \\ Royal College of Art \\ Kensington Gore \\ London SW7 2EU, UK \\ florian.krautli@network.rca.ac.uk, stephen.boyd-davis@rca.ac.uk
}

\begin{abstract}
Historic time is inherently uncertain, with missing, disputed, doubtful or ill-defined dates for objects and events. Yet digital timeline visualisations tend to represent it as exact and of undisputed confidence. This is partly due to a lack of awareness, partly due to a lack of support for ambiguities in digital data types and modes of representation. We outline some of the issues around uncertainty in timeline visualisation and ways of addressing them.
\end{abstract}

Timeline. Uncertainty. Representation. Visualisation.

\section{INTRODUCTION}

Our subject is the visualisation of historical time, including the familiar 'timeline'. We are interested in varied uses of such visualisations: as tools for scholars to support their own thinking by making external representations, perhaps shared with colleagues to promote dialogue concerning historical issues or for communicating to expert or non-expert audiences.

The focus of the present paper is the representation of uncertainty in its many forms (we discuss some of these below). For us, representing uncertainty has two benefits, both connected with a kind of honesty which we believe is pertinent to all of our intended audiences. The most obvious is to be honest about data, in our case data for historical events. We do not want, when making a graphic representation of the available data, to pretend greater certainty than we actually have. As we discuss below, such pretence might involve implying, graphically, that data are known with greater precision than is actually the case, that something disputed is unanimously agreed, or that we have one hundred percent confidence in our source. So, in short, we want to be honest about history. But we also want to be honest about historiography. The majority of visualisations of historic time do two kinds of disservice. They make it look as if each event began and ended with complete certainty at a particular moment in time. But in the process they also present a false picture of what it means to do history. For us, many of the excitements of history lie in doubt and controversy.
Of course it might be objected that all representations simplify, and that the pretended certainty of timelines and other chronographics is just another, uncontentious, simplification. Clearly no representation can pretend to the full subtlety and complexity of the world it represents. Nevertheless, we suggest that the collective 'message' of the great majority of timelines is misleading both about events and history.

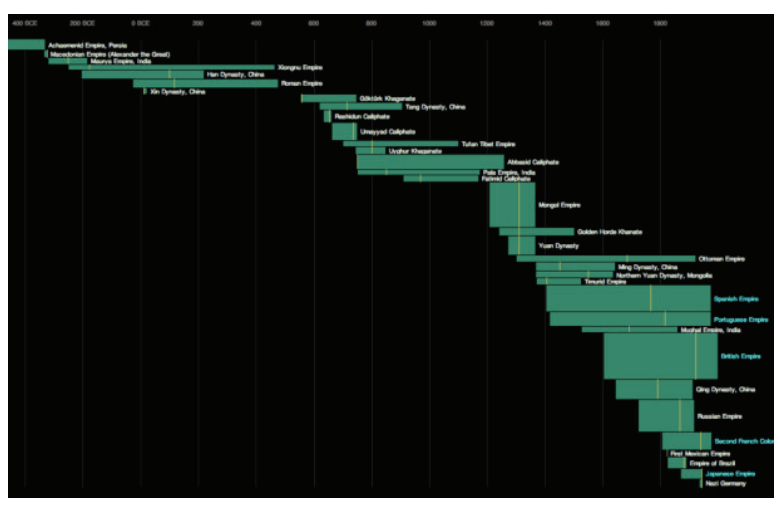

Figure 1: Edward Lee's timeline displays the size and duration of empires as solid blocks. From:

http://visualizing.org/visualizations/historys-largestempires (used with permission)

\section{THE TIMELINE AS DATA VISUALISATION}

Time and timelines are central in the visualisation of cultural data. Understanding the temporal distance, sequence or simultaneity of events is essential when trying to make sense and form a coherent story from a range of individual events or objects. It was this desire to make sense of the 
lives of historic figures that led to the invention of graphical timelines in the eighteenth century (Boyd Davis, 2011). Although timelines today are often designed to be merely illustrative accompaniments to museum exhibitions and written articles, humanities researchers are increasingly making use of data mining and visualisation techniques (Manovich 2007) and the timeline therefore needs to satisfy more rigorous standards of representation. Creators of timelines need to be aware of the problems and errors that can occur when dealing with time, and need to communicate and/or compensate for them appropriately.

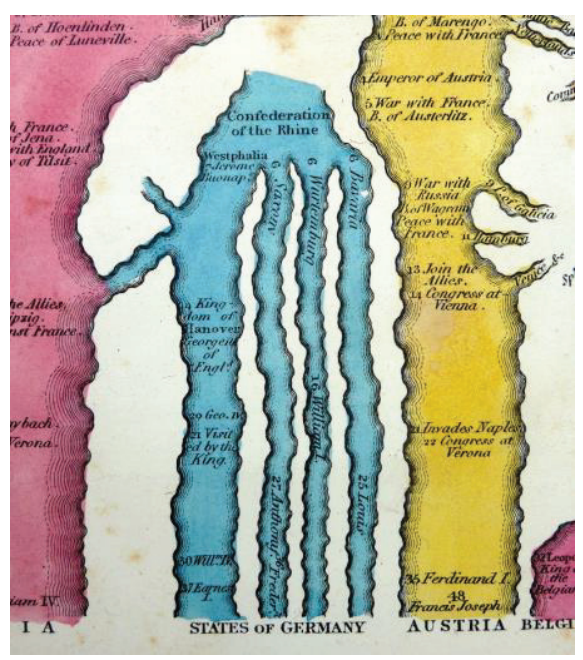

Figure 2: Historic empires flowing in and out of each other. A detail from Strass, F. (1849), Stream of Time (originally Strom der Zeiten, 1804) [London]: C. Smith, Mapseller. Collection: Stephen Boyd Davis. Photo: Stephen Boyd Davis

All data visualisations deal with issues of error and uncertainty and it has been a recurring topic of discussion in recent literature. Dasgupta et al. (2012) propose a taxonomy for visual uncertainty that distinguishes between uncertainties which originate at either the Encoding or Decoding stage. The taxonomy takes on a tree shape which further breaks down into Data Mapping and Visual Mapping on the Encoding branch and Perception and Cognition in the Decoding branch. Pham et al. (2008) study extensively the different levels where uncertainties are introduced and formulate desirable characteristics information visualisation systems need to possess in order to visualise uncertainties honestly. MacEachran et al. (2012) list visual semiotics which may be applied to communicate inaccuracies in data visualisations and present an empirical user study on their individual performance.

Although the representation of errors and uncertainties is standard practice in visualisations of quantitative data, so far little work has been done in addressing the uncertainties in temporal data specifically. Although many lessons learned from numerical data also apply to time, its particular qualities pose some additional problems.

\section{TIME AS DATA}

Transforming time to digital data is not a trivial task, and a range of challenges need to be addressed. Especially when dealing with historic time, dates are often unknown or uncertain. The reasons for this are manifold, some historic facts just can not be known for certain, for others there might exist a range of different recordings all claiming to be true. And even if there is an agreed date recorded, the question remains of how it should be treated if it is in an incompatible, nonGregorian calendar format. Dershowitz and Reingold (2008) list 30 different calendars, including several in current use today and many that are significant in history.

Dating objects or other kinds of cultural production brings up the question of what should be recorded: the period of production, use, the date of sale or resale. Several dates of different meaning might be associated with a single object, but the meaning or reasoning behind the dates is usually not stored within a database. Often the reason for a specific date is found in the biography of its creator or owner. These kind of relations could actually be established through a fitting database architecture, while the data types used to store dates are less flexible and impose great restrictions on how time is recorded.

A common format is the Unix timestamp which expresses a date as the number of seconds passed since the Unix epoch, 1 January 1970 00:00:00. Negative numbers are used to record dates before 1970. For cultural data, the resolution of the digital time format is often much greater than the precision with which historic dates can be known. Dates are stored in the resolution defined by the data type, which may be seconds or days, while the actual date may only be estimated in years or decades. The standard practice is to nevertheless record a complete date (stackoverflow, 2011; 2012), e.g. the first day of the year, if only the year is known. In visual timelines when plotting dates on a linear scale, one might therefore end up with a heap of overlapping data at the beginning of every year. Figure 3 shows this unintentional effect in one of our own timelines. Similarly, if a date range is entered as a way of narrowing down the possible actual date of an event, it needs to be specified as a precise beginning and end date. When representing such an estimate visually, it has to be communicated that the range is in fact an estimate for a single date and not a period of time. The precise nature of digital timelines may 
pretend a level of accuracy not actually supported by the data, an example are the clearly defined periods of empires in Figure 1. Interactive timelines which allow zooming further exaggerate their confidence if zoom levels are not restricted by the granularity of the data.

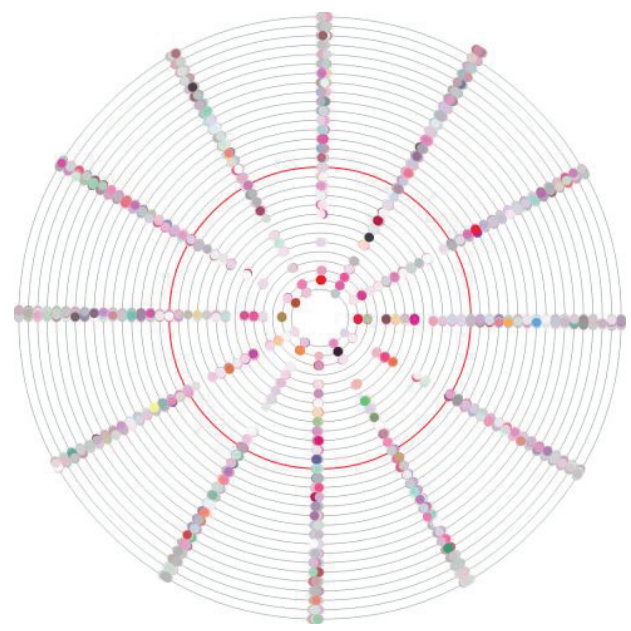

Figure 3: Our experimental tree-ring timeline should reveal seasonaly changing patterns. Instead it reveals quantisation effects in the underlying database architecture designed with the memory limitations of the 1980 s and therefore only storing month and year

This list of challenges is not exhaustive and mainly centres around time in relation to cultural digital data. The digital view on time in particular may have had a big influence on the way we visualise it. Strass's hand-drawn streams of time naturally present a loose and imprecise representation on time, while digital tools foster straightness and precision. Time in the computing sense is strictly linear, discrete and sequential and cannot handle uncertainties, ambiguities in the way that natural language is able to communicate it. The intuitive understanding we have of terms such as "last week" or "around 1940 " cannot so easily be modelled digitally.

When time becomes data, it is often treated as Kosara describes it, as "just another dimension" (Kosara, Bendix, \& Hauser, 2004). On the other hand, he also asserts that "the way we perceive [time] and also its influence on physical phenomena is quite unique". Why then should we not treat time appropriately to its unique qualities and honestly represent its peculiarities?

\section{UNCERTAINTIES IN TIMELINE VISUALISATIONS}

Here we review existing methods and visual models for coping with uncertain descriptions of time in the context of data visualisation.

\subsection{Two pioneers: Priestley and Strass}

Joseph Priestley (1733-1804) dissenting clergyman, educator, scientist and radical, was at one time thought to have invented the modern timeline (Twyman 1986) but more recent work (Ferguson 1991) has discovered a precedent in Barbeu-Dubourg, doctor of medicine, botanist and philologist (these are discussed in Boyd Davis, Bevan and Kudikov 2010). But an innovation of Priestley's is the graphical representation of levels of certainty. Using a drawn line to represent the duration of each life, Priestley identified a problem and offered a solution: 'to express certainty by a full line, and what is uncertain by dots or a broken line' (Priestley 1764: 11). He graded uncertainty into five levels using different numbers of dots.

Using his sparse visual grammar Priestley aims to show transparently what he knows - and does not know. The uncertainties he aimed to mitigate are accidental, arising from uncertain data. A different kind of uncertainty is introduced by designs such as Strass's Stream of Time (see Figure 2) which appeared in many editions through the nineteenth century. As Rosenberg (2007) makes clear, Strass objected to 'the stiff regularity of the straight line' in favour of the stream - 'a common object of sense [that] gives greater liveliness to the ideas, and impresses events more forcibly upon the mind.' This claimed greater rhetorical power comes at the price of aggregating and smoothing discrete events. Authorial imprecision is introduced in the interests of telling a story, a trade-off which still occurs with digital techniques.

\subsection{ThemeRiver}

Havre et al.'s ThemeRiver (Havre et al. 2002) can be seen as a recent counterpart to Strass's Stream of Time. In essence, their visualisation is a reinterpretation of a stacked bar graph which, in their example, presents the occurrences of certain subjects in speeches and articles of Fidel Castro over a period of 40 years, represented as a continuous stream flowing through time, widening when a subject becomes popular, narrowing down or disappearing when a subject is not anymore mentioned. ThemeRiver introduces a visual notation system which makes it easy to follow a pattern through time, although at the cost of losing a level of honesty in the representation. The visualisation draws continuous curves based on discrete data points, there is naturally no factual evidence for every point drawn on the curve. The authors acknowledge this phenomenon. 
If the user zooms in farther than the data resolution supports, our 'truth' as approximated by the interpolated lines is questionable (Havre et al. 2002, p. 3)

The question remains of what the 'truth' means in this context. If 'truth' is regarded as the factual evidence for Castro's interest in a certain topic as recorded in speeches and articles, then indeed ThemeRiver paints a false picture. If by 'truth' we mean Castro's actual interest and concern for the topic, then we deal with measures that can not be known and for which we can not indicate a precise point in time of when they emerged or disappeared. In the latter case the ThemeRiver example is not accurate to the 'truth', but it does represent a more honest picture of what can not be known than a precise graph. We see ThemeRiver as a conceptual model of communicating the uncertainty and interpretability of temporal data.

\subsection{V-model}

The digital timeline has frequently been exploited to visualise and understand medical patient histories. Cousins and Khan (1991) first described the computational and visual model of temporal events and timelines. In doing so, they also recognised the need to address issues of uncertainty and ambiguity in temporal representation (ibid p. 16) as well as the fact that descriptions of temporal events may not necessarily map neatly on to calendar time (ibid p. 15). Plaisant et al. (Plaisant, Milash, Rose, Widoff, \& Shneiderman, 1996) pursued the idea further and developed LifeLines, a visual timeline browser for medical histories. Their model however necessitates events to be explicitly specified within Universal Time.

More recently, Park and Choi (2012) developed V-Model, a new visual model for representing medical histories that breaks with the convention of a linear timeline in order to map both explicit and implicit temporal descriptions. A visual Time Anchoring Point (TAP) relates an event description to a point in time, plotted on a horizontal timeline that lacks the indication of a regular timescale usually found on linear timelines. Instead each TAP is captioned with a temporal expression: an explicit date or time, or an implicit temporal description such as "early 2002", "after Thanksgiving day" or "post-op". TAPs are distributed horizontally and the distance to the preceding TAP depends on the relative temporal distance implied by the temporal descriptions. Gaps between events do not represent absolute periods of time. This design choice allows multiple granularities of time to be represented on the same time axis.
Further work would be necessary to apply a similar visual model also to cultural data. V-Mode is able to represent a single patient's history on a timeline and cope with a certain level of overlapping and simultaneity of events. For mapping cultural data in time it would be necessary to extend the model to deal with several potentially incompatible histories and allow drawing relations between events through compatible TAPs.

\subsection{Temporal Modelling}

Interpreting and determining temporal relations through implicit and uncertain event description is a non-trivial task to work out by an algorithm, as has been shown (Allen 1989; Vilain et al. 1989). Yet it is a common activity when working with cultural data manually and is often performed intuitively and subjectively. Drucker \& Nowviskie (2003) harvest this subjective knowledge in their Temporal Modelling Project by providing an application, dubbed PlaySpace, which allows users to graphically express various kinds of hypothetical temporal expressions and relationships. These individual dictionaries of visual temporal modelling could then be applied in a (theorised) DisplaySpace to generate visualisations based on real data. Ambiguities and uncertainties can be resolved (or not) by moving the position of the user under the surface of the timeline visualisation and allowing the user to interfere with the model that produces it. Resulting visualisations may then not satisfy anymore our need for honesty, taken as an objective notion, as they are subjectively biased. They could however be balanced by combining several models of different users and generating a balanced visual representation.

\subsection{Neatline}

Another project initiated by Nowviskie et al. (2012) worth mentioning is Neatline: an online authoring tool for creating narratives guided by annotated maps and timelines. The timelines are "more-than-usually sensitive to ambiguity and nuance" (Nowviskie et al. 2012) in the sense that they allow a level of ambiguity to be specified for temporal events. Date ambiguity is entered via a range slider, which has two handles to input the ambiguity of the beginning and end date entered. Because the slider always has the same width regardless of the duration of the event in relation to other events on the timeline, it is important to observe the main timeline while dragging the sliders if one aims to maintain a coherent representation of ambiguity across different events of various granularity. Further work would be necessary in the design of the slider to maintain a concise notation of ambiguities 
independent of the author and making such a measure usable for the temporal positioning of cultural data.

\section{WAYS FORWARD}

So far we have presented a range of systems that in different ways respect the uncertain nature of time in their representation of events in timeline visualisations. In the following we will show how these uncertainties emerge and propagate through different stages of the visualisation process and how they can be addressed.

\section{(1) Inherent imprecision of the world}

Dates can be completely unknown or known with a huge range of precision: from centuries through decades, years, down to - in the case of natively digital cultural data - minutes and seconds.

\section{(2) Interpretation by curator}

A curator will give an estimate for unknown dates or resolve conflicting accounts of history by giving preference to one or reconciling them. If dates are not recorded explicitly but e.g. through textual descriptions, they need to be interpreted. A curator of cultural data always adds his or her own interpretation.

\section{(3) Input through an user interface}

Most database management tools we have seen offer an input mask for data which is a reflection of its underlying architecture. Dates are entered through a date input field and expect the input to be compatible with the data types.

\section{(4) Interpretation by system}

When new data is entered into a database system it needs to be sanitised in order to ensure compatibility with the data type. The amount of system-side interpretation depends on the level of ambiguity allowed in the input layer. For example, if the data type expects a date to consist of year, month and day, the system needs to add missing information in case only a year is provided.

\section{(5) Visual representation}

A visual representation is always a translation which introduces another potential for errors. On one hand through the graphical mapping itself, on the other hand through presenting a level of confidence that is not actually in the data.

We will address (3) and (4) in relation to the data model and (5) which involves the visual model.

\subsection{Uncertain times in data models (3) (4)}

We have observed a lack of support for uncertainties in time within the architecture of database models and consequently in the user interface for entering data. In order to visualise temporal uncertainty honestly, it is essential to have such information recorded. Such recordings are very rarely found in current collections and therefore need to be assumed. When building new collections, the chance of collecting confidence data as outlined in the following steps should not be missed.

\subsubsection{Measure of confidence}

Neatline (Nowviskie et al. 2012), above, features a range slider to specify one's subjective confidence of beginning and end dates. The measure directly affects the visual encoding while also being stored in the database. We could also imagine less fine-grained measures which may counteract the subjective judgement towards a more concise notation of confidence.

\subsubsection{Restrictions and relations}

Most current database models support establishing various kinds of relations between database records, yet time is usually treated as an explicit measure rather than a relative statement. The temporal ordering of events in digital systems is well understood (Lamport 1978) and database models should support the temporal positioning of events both explicitly as well as implicitly through the establishment of 'before', 'after' or 'during' relations.

\subsubsection{Renounce calendars}

Forcing users to specify a date in the calendar format obliges them to express a belief with higher precision than they are actually able to. For example, a statement such as 'in the 1930s' is implicitly understood in natural language, but does not necessarily mean to translate into 1 January 1930 - 31 December 1939. If the calendar is skipped users can enter their original belief directly in natural language. Apple's Siri (Apple Inc., 2011) as well as the calendar software Fantastical (Flexibits 2011) already allow sophisticated scheduling of events in natural language. Even if the inputs are still interpreted to calendar dates, there are inherent advantages if the original input is stored along with the interpretation: if this meta data is exposed at the visualisation level, it may guide the user in properly interpreting the explicit data. 


\subsection{Uncertain times in visual models (5)}

A data visualisation should represent its underlying data as accurately as possible. In the case of timelines however, being true to the data does not necessarily mean being honest to history as current data types are often not able to properly represent uncertainties and ambiguities.

\subsubsection{Assume uncertainty, respect granularity}

Even without an indication of confidence in the data, it can often be inferred from the range and granularity with which events and objects are dated. A visualisation should not take such temporal data at face value, but indicate possible uncertainties and not represent data at finer granularity than it is specified in.

\subsubsection{Visualise dependencies and relations}

A timeline should reveal the succession of events and provide context that enable the circumstances around events to be understood in their current time. It is much easier to relate to temporal landmarks than to abstract dates (Ringel, Cutrell, Dumais, \& Horvitz, 2003). Continuum (André et al. 2007) uses a hierarchical format to visualise compositions related to the lifetime of their composers. The relative temporal position of the composition towards its author is more informative than a precise date.

\subsubsection{Consider non-linear or non-calendric time formats}

Linear and universal time are not the only possible models for visualising time. If one is interested in the precise dates of events, a list may actually serve better than a timeline. The advantage of visualising time is in seeing relations, successions, sequence and simultaneity, all of which can be shown without the need of anchoring all events in universal time. As has been demonstrated by V-Model, not enforcing explicit dates does not necessarily mean compromising on honesty.

\subsection{Uncertain times in combining data and visual models (3) (4) (5)}

We present our own attempts at developing a combined data type and visual model to both record and represent uncertainties in historic data. We are developing methods, which allow the computational modelling of uncertainties in a way that can be translated directly to a visual representation. We do not intend to offer a complete solution, but aim to encourage alternative accounts of time recording and representing in order to facilitate more honest digital timelines. Our model borrows from probability theory as it provides a formal language for expressing uncertainties that can be understood in computational terms as well as being translated to a visual representation.

\subsubsection{Uncertain times as probabilities}

Estimating the date of an object or event means expressing a level of confidence towards a certain date or timeframe associated with it. Stating that a cup has been manufactured around 1967 conveys a high level of confidence that it has been made at some time between 1965 and 1969, but does not completely rule out the possibility of it being made in 1961 or even 2001. If we record this statement as 1965 - 1969 precisely, which we need to do with many current data types, we set clear boundaries which may not be true and lose the original uncertainty inherent in the statement 'around 1967'. Some circumstances may allow a timeframe to be defined more explicitly, such as if the year of production is marked on an object. In this example, we have a consistent level of confidence that the cup has been made during the year 1967, and we may completely rule out the possibility of it having been made outside of this timeframe.

If we want to store these kind of estimates in a conventional discrete format, we need to answer the question of when 'exactly' an object has been manufactured, which we are naturally unable to know simply by inspecting it. We may however be able to answer how likely it is, that an object has been manufactured at some time in 1967. Formally, these questions can be answered by a probability density function ( $p d f)$, which is the format we propose in which ambiguous temporal statements may be recorded. A pdf is a function which models the likelihood of a random variable to be in a given range. The probability is defined by the integral of the function over the given period, which means we always need to specify a period of time rather than a point in time. The probability of an event having taken place at an exact point in time is always zero, which reflects our idea of what we are able to know.

We will introduce two commonly used pdf's to model our two example statements. For reasons of readability, we will note all dates as years, but they could be in any other format (e.g. Unix time). 'Around 1967' can be modelled as a normal distribution with a mean of 1967 and a standard deviation of 1 . The deviation may be guessed by the system or selected by the user. 'Around 1967' implies a certain range of confidence, as does e.g. '18th century', which implies a greater range. As a pdf our example statement reads:

$$
f(x)=\frac{1}{\sqrt{2 \pi}} e^{-\frac{(x-1967)^{2}}{2}}
$$


This function returns a probability of around 95\% that the event happened some time between 1965 and 1969, which raises to $99.7 \%$ if we extend the range by one year on each side. Note that there is still a $0.3 \%$ probability that the event took place outside of this timeframe. For the more defined case of 1967 we model the statement as a uniform pdf over the period of the entire year, which will produce a probability of zero outside of the given timeframe and a constant probability for all values within:

$$
f(x)=\left\{\begin{array}{l}
1 \text { for } 1967 \leq x \leq 1968 \\
0 \text { for } x<1967 \text { or } x>1968
\end{array}\right.
$$

\subsubsection{Towards a visual representation}

If we now plot both functions as in Figure 4, using the $\mathrm{x}$-axis for time we arrive at a visual representation which is directly derived from the data model and is able to convey information both about the described timeframes as well as their relative ambiguity.

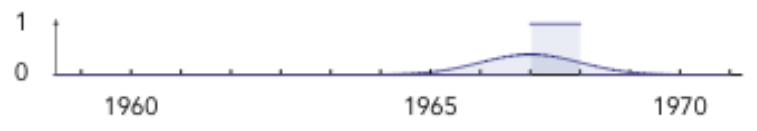

Figure 4: Function plots of two probabilistic time descriptions

a)

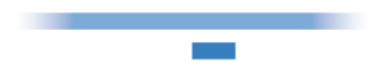

b)

c) 〜 unow

Figure 5: Possible renderings of probabilistic time descriptions as timeline events

We do not suggest using this representation directly in a visual timeline as it can easily be misread for a measure of quantity rather than certainty. Instead we propose to render the functions in a visual language that borrows from familiar conventions. Figure $\mathbf{5}$ presents three possibilities of rendering the functions as events on a timeline. We applied some thresholding and let the functions control opacity (a), line dashing (b) and wavelength (c), some of the visual parameters for uncertainty we explored in our earlier work (Boyd Davis et al. 2010). Controlling other parameters, such as the ones described by MacEachren (2012) or different kind of visual or even non-visual renderings could be explored.

\subsubsection{Drawbacks}

The major drawback of modelling dates as continuous functions instead of discrete ranges is that it is computationally much more expensive. The cost depends on the other hand also on the resolution at which the functions are processed. In the above example we applied the thresholding not only in order to make the representation more readable, but also to simplify its calculation. If the events are represented individually, the values can also be simulated as they are standard functions controlled by only a few parameters. When events are processed combined, the functions can be approximated up to the resolution necessary.

Another issue worth considering is the improper visual interpretation of pdfs, which may introduce another source of error. It is essential that the modelling and effect of the functions are properly applied and understood.

\section{CONCLUSION}

In our claim for more honest digital timelines we have outlined the inherent uncertainties of time on one hand and the lack of consideration and support for storing and representing uncertainties in cultural data and visualisations on the other. The awareness of uncertainties which early timelines exhibited is rarely found in current digital timelines, with the exception of a few cases we have presented. From those examples as well as our own work, we derived guidelines on how to deal with uncertain temporal data both in the visualisation of legacy data, as well as in the acquisition of new cultural data. There are many opportunities for innovation, both theoretically and through designing.

\section{REFERENCES}

Allen, J. F. (1989). Maintaining knowledge about temporal intervals. Readings in qualitative reasoning about physical systems.

André, P., Wilson, M. L., Russell, A., Smith, D. A., Owens, A., \& schraefel, M. C. (2007). Continuum: designing timelines for hierarchies, relationships and scale. UIST '07: Proceedings of the 20th annual ACM symposium on User interface software and technology.

Apple Inc. (2011). Apple Siri. apple.com. Retrieved April 1, 2013, from http://www.apple.com/ios/siri/

Boyd Davis, S. (2011). Joseph Priestley: The Man Who Drew Time. Postings from the Priestley House. 
Boyd Davis, S., Bevan, E., \& Kudikov, A. (2010). Just in time: defining historical chronographics. Presented at the EVA'10: Proceedings of the 2010 international conference on Electronic Visualisation and the Arts, British Computer Society, London.

Cousins, S. B., \& Kahn, M. G. (1991). The visual display of temporal information. Artificial intelligence in medicine, 3(6), 341-357.

Dasgupta, A., Chen, M., \& Kosara, R. (2012). Conceptualizing Visual Uncertainty in Parallel Coordinates, 31(3pt2), 1015-1024.

Dershowitz, N. and Reingold, E.M. (2008) Calendrical Calculations 3rd ed. (Cambridge University Press, New York).

Drucker, J., \& Nowviskie, B. P. (2003). Temporal Modelling: Conceptualization and Visualization of Temporal Relations for Humanities Scholarship. ACH/ALLC 2003 Conference, 26-28.

Ferguson, S. (1991) The 1753 Carte Chronographique of Jacques Barbeu-Dubourg. Princeton University Library Chronicle. (Winter 1991).

http://www. princeton.edu/ ferguson/PULC_1991_ duBourg.pdf (Accessed 3 April 2013).

Flexibits. (2011). Fantastical for Mac. flexibits.com. Retrieved April 1, 2013, from http://flexibits.com/fantastical

Havre, S., Hetzler, E., Whitney, P., \& Nowell, L. (2002). Themeriver: Visualizing thematic changes in large document collections. Visualization and Computer Graphics, IEEE Transactions on, 8(1), 9-20.

Kosara, R., Bendix, F., \& Hauser, H. (2004). Timehistograms for large, time-dependent data. Symposium on Visualization, 45-54.

Lamport, L. (1978). Time, clocks, and the ordering of events in a distributed system. Communications of the ACM, 21(7), 558-565.

MacEachren, A. M., Roth, R. E., \& O'Brien, J. (2012). Visual Semiotics \& Uncertainty Visualization: An Empirical Study. IEEE Transactions on Visualization and Computer Graphics.

Manovich, L. (2007, September). Cultural Analytics: Analysis and Visualization of Large Cultural Data Sets. manovich.net. Retrieved March 7, 2013, from http://www.manovich.net/cultural_analytics.pdf
Nowviskie, B. P., McClure, D., Graham, W., Rochester, E., \& Boggs, J. (2012). Neatline.org | About. neatline.org. Retrieved March 5, 2013, from http://neatline.org/about/

Park, H., \& Choi, J. (2012). V-model: a new innovative model to chronologically visualize narrative clinical texts, 453-462.

Pham, B., Streit, A., \& Brown, R. (2008). Visualisation of Information Uncertainty: Progress and Challenges. In Advanced Information and Knowledge Processing (pp. 19-48). London: Springer London.

Plaisant, C., Milash, B., Rose, A., Widoff, S., \& Shneiderman, B. (1996). LifeLines: visualizing personal histories. $\mathrm{CHI}$ '96: Proceedings of the SIGCHI conference on Human factors in computing systems: common ground, 221-227.

Priestley, J. (1764) A Description of a Chart of Biography. Warrington. British Library General Reference Collection 10604.aa.11.

Ringel, M., Cutrell, E., Dumais, S., \& Horvitz, E. (2003). Milestones in time: The value of landmarks in retrieving information from personal stores. In G. W. M. Rauterberg, M. Menozzi, \& J. Wesson (Eds.), Human-Computer Interaction, Interact '03: (Vol. 2003, pp. 184-191).

Rosenberg, D. (2007) Joseph Priestley and the Graphic Invention of Modern Time. Studies in Eighteenth Century Culture 36(1). 55-103.

stackoverflow. (2011). Storing day and month (without year) stackoverflow.com. Retrieved April 2, 2013, from http://stackoverflow.com/questions/4998470/storin g-day-and-month-without-year

stackoverflow. (2012). mysql datatype to store month and year only stackoverflow.com. Retrieved April 2, 2013, from http://stackoverflow.com/questions/9134497/mysq I-datatype-to-store-month-and-year-only

Twyman, M. (1986) Articulating Graphic Language: a historical perspective. In Wrolstad, M.E. and Fisher, D.F. Towards a New Understanding of Literacy. Praeger, New York. 188-251.

Vilain, M., Kautz, H., \& van Beek, P. (1989). Constraint propagation algorithms for temporal reasoning: a revised report. Readings in qualitative reasoning about physical systems. 